\title{
CLASIFICACIÓN DE LAS COMUNIDADES ACUÁTICAS DEL SECTOR CELTIBÉRICO-ALCARREÑO (CENTRO DE LA PENÍNSULA IBÉRICA)
}

\author{
José A. MOLINA y Santiago SARDINERO
}

\begin{abstract}
RESUMEN. Clasificación de las comunidades acuáticas del sector Celtibérico-Alcarreño (centro de la Península Ibérica). Este trabajo, combinando el método fitosociológico de Braun-Blanquet con análisis numéricos de agrupamiento y ordenación, describe la vegetación acuática de los territorios situados en la submeseta norte calcárea de la Península Ibérica, biogeográficamente pertenecientes al sector CeltibéricoAlcarreño (provincia Castellano-Maestrazgo-Manchega). Se han identificado las siguientes asociaciones y comunidades: Comunidad de Chara hispida, Groenlandio densae-Zannichellietum peltatae, Potametum colorati, Hippuridetum vulgaris, Comunidad de Polygonum amphibium, Potamo pectinati-Myriophylletum spicati y Myriophyllo-Nupharetum lutei.
\end{abstract}

Palabras clave. Análisis multivariante, Charetea, Clasificación, España, Fitosociología, Ordenación, Potametea, Sintaxonomía.

ABSTRACT. Classification of aquatic plant-communities of the Celtiberico-Alcarreño sector (central Iberian Peninsula). This study, combining the Braun-Blanquet phytosociological approach with numerical methods (cluster and ordination analysis), describes the aquatic vegetation of the calcareous upper Iberian Plateau belonging to the Celtiberico-Alcarreño biogeographical sector (Castellano-Maestrazgo-Manchega province). The following associations and communities have been identified: Community of Chara hispida, Groenlandio densae-Zannichellietum peltatae, Potametum colorati, Hippuridetum vulgaris, Community of Polygonum amphibium, Potamo pectinati-Myriophylletum spicati and MyriophylloNupharetum lutei.

Key words. Charetea, Classification, Multivariate analysis, Ordination, Phytosociology, Potametea, Spain, Syntaxonomy.

\section{INTRODUCCIÓN Y OBJETIVOS}

En la Península Ibérica existen numerosos estudios sobre vegetación de humedales tanto litorales como interiores (Cirujano, 1980; Rivas-Martínez et al., 1980; Asensi y Nieto, 1981; Arnaiz y Molina, 1986; Costa et al.,
1986; Rodriguez Oubiña, 1986; Velayos et al., 1989; Pérez Raya y López Nieto, 1991; Biurrun Galarraga, 1995; Bueno Sánchez, 1997). Sin embargo, la vegetación acuática de los territorios meridionales calizos de la submeseta norte no había sido tratada, hasta ahora, más que de modo marginal (Velayos et al., 1984; 
Molina, 1996). El objetivo de este trabajo se centra en la clasificación y descripción de las comunidades acuáticas incluibles en las clases fitosociológicas Charetea fragilis y Potametea, que se desarrollan sobre sustratos ricos en bases de las tierras altas de Cuenca y Guadalajara, en el centro de la Península Ibérica.

\section{ÁREA ESTUDIADA}

El área estudiada se situa en el centro de la Península Ibérica, entre $1^{\circ} 30^{\prime}$ - $3^{\circ} 10^{\prime}$ Oeste y $40^{\circ} 10^{\prime}-41^{\circ} 20^{\prime}$ Norte, y entre los 750 y 1280 $\mathrm{m}$ de altitud (fig. 1). Practicamente todas las localidades muestreadas se ubican, dentro de la provincia Castellano-Maestrazgo-Manchega, en el sector Celtibérico-Alcarreño el cual ocupa las alcarrias y parameras de Guadalajara y Cuenca (tab. 1). De acuerdo con Rivas-Martínez (1987), la vegetación potencial de la mayor parte del territorio pertenece a la serie supramesomediterránea castellano-alcarreñamanchega basófila de Quercus faginea o quejigo, Cephalantero longifoliae-Querceto fagineae sigmetum.

El área centro-oriental de la Península Ibérica comparte dos factores desfavorables

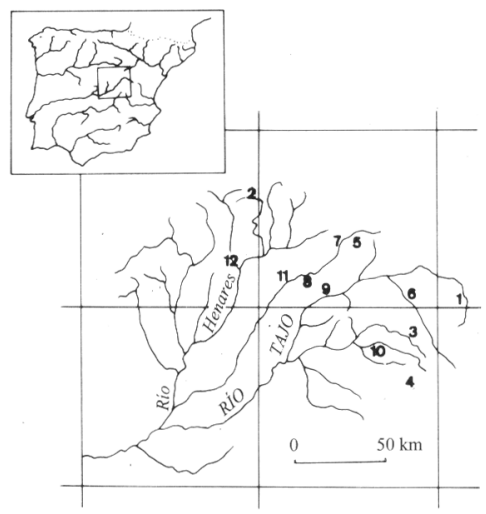

Figura 1. Situación geográfica del área de estudio y localidades muestreadas. Geographical situation of the study area and sampling localities. para la existencia de complejos palustres permanentes. Por una parte, está sometido a un clima mediterráneo con acusada sequía estival. Por otra, constituye una región calcárea en la que las formaciones superficiales de agua están dificultadas debido a la porosidad de la roca. En los territorios calizos estudiados, el sistema fluvial viene fundamentalmente condicionado por la red hidrográfica del río Tajo y, marginalmente, por la del río Júcar, ambas de orientación E-O. La expresión lacustre más común es el lago kárstico de aguas dulces y permanentes, mineralizadas y de turbidez variable por eutrofía (Alonso, 1987).

\section{MÉTODOS Y TERMINOLOGÍAS}

La toma de datos fue realizada durante los años 1989 a 1991, utilizando el método de muestreo por replicación centralizada (MuellerDombois \& Ellenberg, 1974). Las parcelas de muestreo fueron elegidas de acuerdo con los biotipos y las formaciones fisionómicoecológicas reconocidas (Hartog \& Segal, 1964).

El estudio se ha basado en una matriz, compuesta por 50 inventarios y 24 plantas, ordenados en un tabla de acuerdo con Westhoff \& van der Maarel (1978) y Braun-Blanquet (1979). Los valores de abundancia/dominancia en la escala de Braun-Blanquet fueron transformados en la escala propuesta por van der Maarel (1979). La clasificación de los inventarios fue realizada mediante técnicas de agrupamiento secuencial, aglomerante, jerárquico y no solapante (Sneath \& Sokal, 1973; Podani, 1989). Se utilizó la razón de similitud, la distancia de la cuerda y la distancia euclídea, para el cálculo de la distancia entre cada par de inventarios dentro de la matriz de semejanzas. Los inventarios fueron agrupados mediante vinculación completa, y minimización de la suma del cuadrado de las desviaciones con respecto a su centroide, utilizando el programa de ordenador NCLAS 


\begin{tabular}{llcccc}
\hline & Localidad, provincia, U.T.M. & Altitud (m) & Cuenca hidrográfica & Piso bioclimático & Vegetación natural potencial \\
\hline 1 & Laguna de las Majanas, Setiles, Gu, 30TXL11 & 1280 & Tajo & Supramediterráneo & Cephalantero longifoliae-Quercetum fagineae \\
2 & Laguna de Somolinos, Gu, 30TVL96 & 1250 & Tajo & Supramediterráneo & Juniperetum hemisphaerico-thuriferae \\
3 & Lagunas de El Tobar, Beteta, Cu, 30TWK88 & 1150 & Tajo & Supramediterráneo & Cephalantero longifoliae-Quercetum fagineae \\
4 & Laguna de Uña, Cu, 30TWK85 & 1140 & Júcar & Supramediterráneo & Cephalantero longifoliae-Quercetum fagineae \\
$\mathbf{5}$ & Laguna de la Hortezuela de Océn, Gu, 30TWL53 & 1130 & Tajo & Supramediterráneo & Cephalantero longifoliae-Quercetum fagineae \\
$\mathbf{6}$ & Laguna de Taravilla, Gu, 30TWL80 & 1120 & Tajo & Supramediterráneo & Cephalantero longifoliae-Quercetum fagineae \\
7 & Río Tajuña, Luzaga, Gu, 30TWL43 & 1050 & Tajo & Supramediterráneo & Cephalantero longifoliae-Quercetum fagineae \\
8 & Río Tajuña, Masegoso de Tajuña, Gu, 30TWL21 & 875 & Tajo & Supramediterráneo & Cephalantero longifoliae-Quercetum fagineae \\
9 & Río Cifuentes, Gárgoles de Abajo, Gu, 30TWL30 & 850 & Tajo & Supramediterráneo & Cephalantero longifoliae-Quercetum fagineae \\
10 & Río Escabas, Priego, Cu, 30TWK67 & 830 & Tajo & Supramediterráneo & Cephalantero longifoliae-Quercetum fagineae \\
11 & Río Tajuña, Brihuega, Gu, 30TWL11 & 810 & Tajo & Supramediterráneo & Cephalantero longifoliae-Quercetum fagineae \\
12 & Río Sorbe, Cogolludo, Gu, 30TVL82 & 750 & Tajo & Mesomediterráneo & Cephalantero longifoliae-Quercetum fagineae \\
\hline
\end{tabular}

Tabla 1. Datos geográficos y geobotánicos de las localidades estudiadas. Geographical and geobotanical data of the surveyed localities.

(Podani, 1993). Las unidades de vegetación definidas mediante el análisis de agrupamiento fueron superpuestas a diagramas de ordenación obtenidos mediante análisis de correspondencias (CA) llevados a cabo con el programa CANOCO (ter Braak, 1985; 1990), con el objetivo de conseguir un análisis más completo de los grupos, y de analizar las relaciones entre vegetación y flora. Los grupos obtenidos fueron considerados asociaciones florísticas y en la mayor parte de los casos fueron incluidos en asociaciones fitosociológicas descritas.

La nomenclatura de las plantas vasculares sigue a Tutin et al. (1964-1980). La tipología biogeográfica se halla de acuerdo con Peinado Lorca y Martínez Parras (1987) y RivasMartínez et al. (1990). La tipología sintaxonómica aceptada se muestra en el esquema sintaxonómico anejo. En las series de vegetación se sigue a Rivas-Martínez (1987).

\section{RESULTADOS Y DISCUSIÓN}

\section{Clasificación}

Los inventarios fueron dispuestos en la tabla 2, de acuerdo con el orden sugerido por el dendrograma que se obtuvo mediante razón de similitud y vinculación completa (fig. 2 a). Con otras clasificaciones se obtuvieron resultados similares (fig. 2b; fig. 2c; fig. 2d). De este modo, los inventarios fueron reunidos en 7 grupos. De acuerdo con el sentido de Font Quer (1982), uno de ellos (grupo A) corresponde a la holohydrophytia adnata (clase Charetea) mientras que los demás (grupos B, C, D, E, F y G) corresponden a la vegetación propia de la holohydrophytia radicantia (clase Potametea).

El grupo A (tab. 2, inv. 1-6) está constituido por los inventarios dominados por Chara hispida. Este grupo constituye el primer cinturón de vegetación sobre suelos limneos en buena parte de las lagunas kársticas del territorio; en la sucesión natural es reemplazado hacia los márgenes menos profundos por los rizófitos acuáticos. Dentro de este grupo, se ha advertido un subgrupo con Potamogeton natans en el que la comunidad se presenta estructurada en dos estratos: uno formado por un tapiz de haptófitos sumergido y arraigado al fondo, y otro, cormofítico, enraizado y con hojas flotantes. En el interior peninsular se ha descrito la asociación Charetum conniventis de aguas temporales que incluye en su composición florística Chara canescens en aquellas localidades con mayor salinidad (Velayos et al., 1989). Las diferencias ecológicas (menor permanencia del agua) y florísticas (presencia de Chara connivens) de esta asociación manchega frente a las comunidades celtibéricoalcarreñas estudiadas, justifican la 


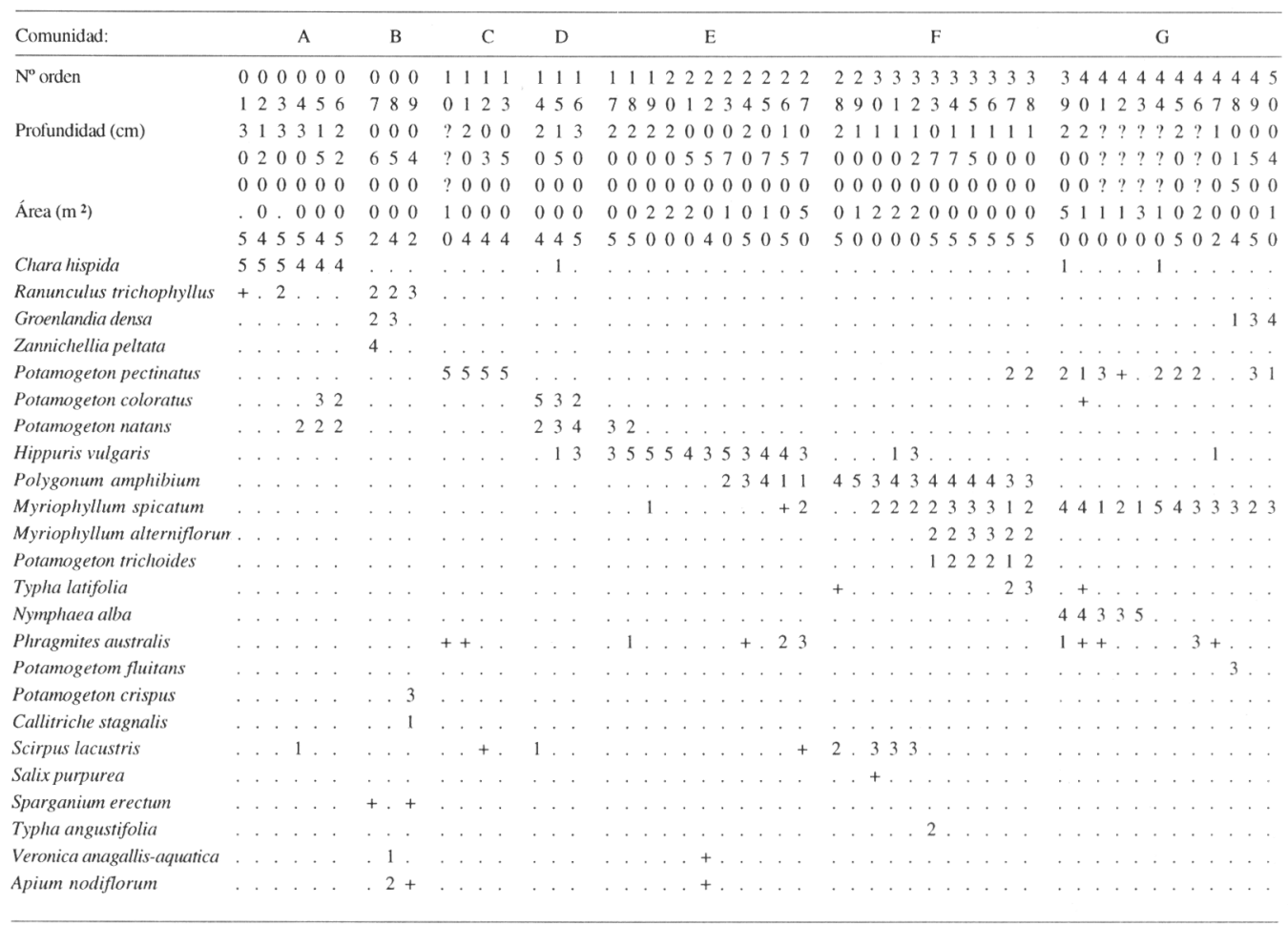

Tabla 2. Tabla fitosociológica de las comunidades acuáticas del sector Celtibérico-Alcarreño. Comunidades: A, Comunidad de Chara hispida; B, Groenlandio densae-Zannichellietum peltatae; C, Potamo pectinatiMyriophylletum spicati; D, Potametum colorati; E, Hippuridetum vulgaris; F, Comunidad de Potamogeton amphibium; G, Myriophyllo-Nupharetum lutei. Localidades (entre paréntesis figura la fecha-día, mes, dos últimas cifras del año- y un número de orden): 01, Laguna de Somolinos (2098907); 02, Laguna de Somolinos (2906901); 03, Laguna de Somolinos (2098907); 04, Laguna de Taravilla (1310892); 05, Laguna de Taravilla (3006901); 06, Laguna de Taravilla (3006901); 07, Laguna de Somolinos (2906901); 08, Río Escabas, Priego (1307904); 09, Río Cifuentes, Gárgoles de Abajo (1007904); 10, Laguna Grande de El Tobar (1609891); 11, Laguna de la Hortezuela de Océn (1007905); 12, Río Tajuña, Brihuega (1007902); 13, Río Tajuña, Masegoso de Tajuña (1007903); 14, Laguna de Taravilla (3006901); 15, Laguna de Taravilla (3006901); 16, Laguna de Taravilla (3006901); 17, Laguna de Taravilla (3006901); 18, Laguna de Taravilla (1310892); 19, Laguna de Uña (1110891); 20, Laguna de Uña (1508901); 21, Laguna de Uña (1508901); 22 , Laguna de Uña (1508901); 23, Laguna de Uña (1508901); 24, Laguna de Uña (1110891); 25, Laguna de Uña (1508901); 26, Laguna de Uña (1110891); 27, Laguna de Uña (1508901); 28, Laguna de Uña (1110891); 29, Laguna de Uña (1508901); 30, Laguna de Uña (1508901); 31, Laguna de Uña (1508901); 32, Laguna de Uña (1508901); 33, Laguna de las Majanas, Setiles (1207902); 34, Laguna de las Majanas, Setiles (1207902); 35, Laguna de las Majanas, Setiles (1207902); 36, Laguna de las Majanas, Setiles (1207902); 37, Laguna de las Majanas, Setiles (1207902); 38, Laguna de las Majanas, Setiles (1207902); 39, Laguna Grande de El Tobar (1609891); 40, Laguna Pequeña de El Tobar (1609892); 41, Laguna Grande de El Tobar (1609891); 42, Laguna Grande de El Tobar (1609891); 43, Laguna Grande de El Tobar (1609891); 44, Laguna Grande de El Tobar (1609891); 45, Laguna de las Majanas, Setiles (1207902); 46, Laguna Grande de El Tobar (1609891); 47, Laguna de Uña (1110891); 48, Río Sorbe, Cogolludo (907905); 49, Río Tajuña, Luzaga (1210891); 50, Río Tajuña, Luzaga (1210891). Phytosociological table of the aquatic plant-communities in the Celtiberico-Alcarreño sector. 

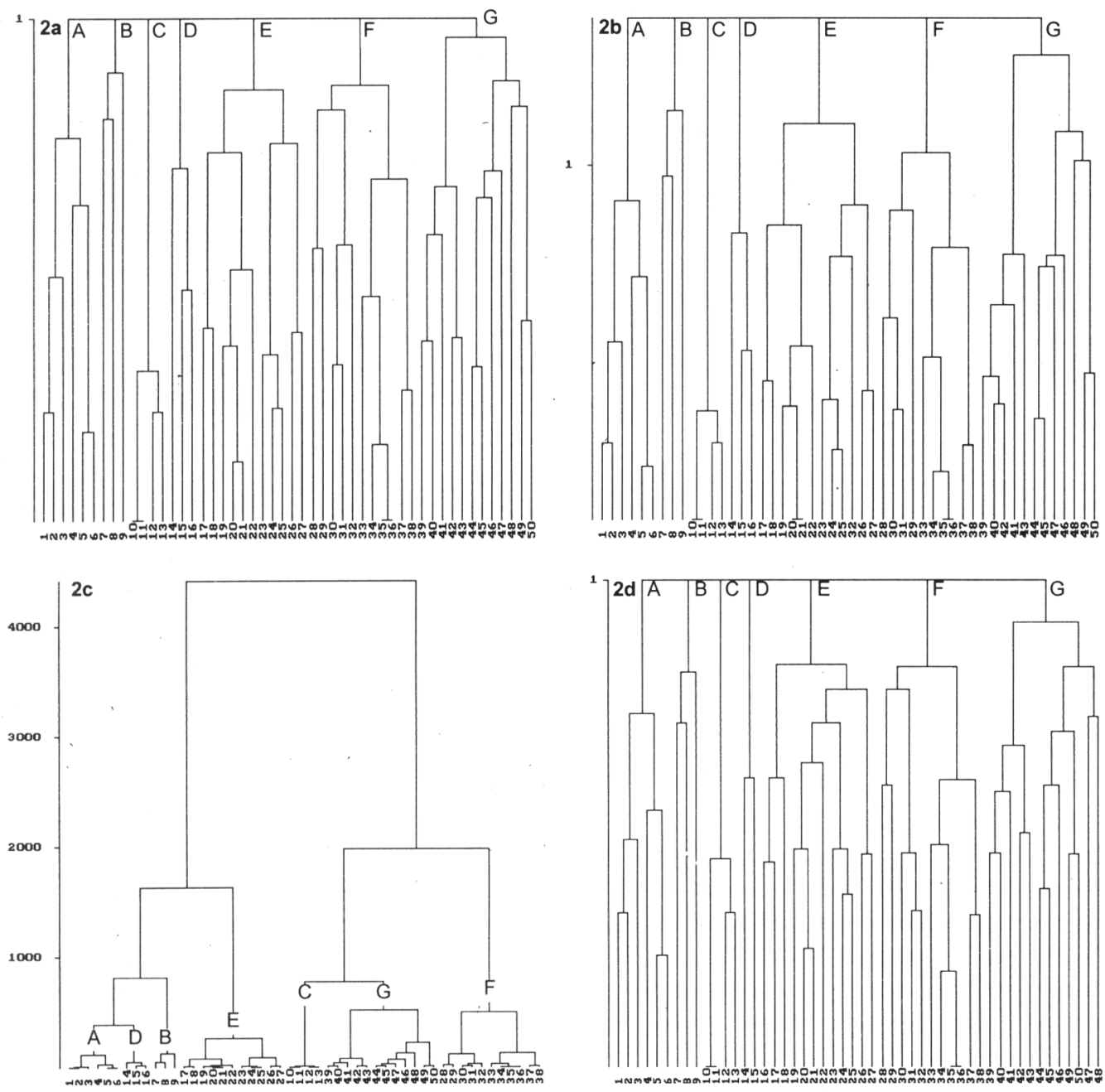

Figura 2. Clasificación secuencial, aglomerante, jerárquica y no solapante mediante: razón de similitud y vinculación completa (2a); distancia de la cuerda y vinculación completa (2b); distancia euclídea y minimización de la suma del cuadrado de las desviaciones (2c); porcentaje de diferencia y vinculación completa (2d). Grupos: A, Comunidad de Chara hispida; B, Groenlandio densae-Zannichellietum peltatae; C, Potamo pectinati-Myriophylletum spicati; D, Potametum colorati; E, Hippuridetum vulgaris; F, Comunidad de Potamogeton amphibium; G, Myriophyllo-Nupharetum lutei. Sequential, agglomerative, hierarchical and non-overlapping classification using: similarity ratio and complete linkage-CL-(2a); chord distance and $C L(2 b)$; Euclidean distance and minimum increase of the error sum of squares (2c); percentage difference and $C L(2 d)$. 
independencia sintaxonómica entre ambas.

El grupo B (tab. 2, inv. 7-9) incluye los inventarios dominados por pequeños elodeidos y batráchidos de bordes de aguas someras y fluyentes. Este grupo se ha asimilado a la asociación Groenlandio densaeZannichellietum peltatae descrita en el sector Celtibérico-Alcarreño (Velayos et al., 1989). Catenalmente contacta con comunidades adjudicables a la asociación Potametum densonodosi O. Bolòs 1957 de las que no se ha podido aportar ningún inventario. Este grupo guarda asímismo afinidades florísticas y ecológicas evidentes con la asociación Ranunculo trichophylli-Groenlandietum densae (Kohler et al., 1974) Passarge 1994 reconocida en el centro-septentrional de España (Loidi Arregui et al., 1997).

El grupo C (tab. 2, inv. 10-13) reúne los inventarios de elodeidos de hojas filiformes que se desarrollan en aguas oxigenadas (ríos o desembocaduras/desagües de lagunas) y relativamente profundas. Se trata de una comunidad pauciespecífica dominada por Potamogeton pectinatus y acompañada a lo sumo por algún helófito. Adscribimos los inventarios aportados a la asociación Potamo pectinati-Myriophylletum spicati descrita en aguas mineralizadas subsalinas de la cuenca del Guadiana, y caracterizada por Myriophyllum spicatum, Potamogeton pectinatus, Ranunculus trichophyllus y Potamogeton fluitans (Rivas Goday, 1964). Otras asociaciones próximas son Potametum pectinati descrita en el litoral holandés y caracterizada por Potamogeton pectinatus var. scoparius (Carstensen, 1955), y Myriophyllo verticillati-Potametum pectinati descrita en el levante español y caracterizada por Potamogeton pectinatus y Myriophyllum verticillatum (Costa et al., 1986).

El grupo D (tab. 2, inv. 14-16) constituye los inventarios dominados por elodeidos y pequeños ninfeidos de márgenes profundos de aguas quietas. Este grupo, detectado en una sóla localidad (laguna de Taravilla), está floristicamente caracterizado por Potamogeton coloratus y Potamogeton natans. Sintaxonómicamente se incluye en la asociación Potametum colorati.

El grupo E (tab. 2, inv. 17-27) agrupa los inventarios dominados por Hippuris vulgaris. Dentro de él, puede reconocerse, una variante local con Polygonum amphibium en la laguna de Uña (inv. 23-37). Este grupo se ha adscrito a la asociación Hippuridetum vulgaris que algunos autores europeos (Philippi, 1974; Pott, 1992) enmarcan en la clase fitosociológica Phragmito-Magnocaricetea pero que en este trabajo se ha incluido en Potametea ya que el biotipo que muestra Hippuris vulgaris en las comunidas estudiadas es el acuático de elodeido y no el terrestre de helófito. Hippuris vulgaris muestra en la Península Ibérica una distribución Castellano-Maestrazgo-Manchega y Pirenáica (Penas Merino y Díaz González, 1985) por lo que la asociación probablemente tenga esa distribución.

El grupo F (tab. 2, inv 28-38) incluye inventarios relativamente heterogéneos - dominados por Polygonum amphibium, con biotipo ninfeido. En este grupo se han diferenciado dos subgrupos, uno que corresponde a los inventarios de la laguna de Uña, y otro en la laguna de las Majanas más diversa en número de especies sobre todo de los biotipos elodeido y miriofílido, y de aguas menos básicas. Las comunidades dominadas por Polygonum amphibium han recibido un tratamiento sintaxonómico muy diverso debido, probablemente, a la amplia valencia ecológica de este taxon. Meriaux (1978) ha considerado ciertas comunidades de Polygonum amphibium en el norte de Francia como facies de la asociación Myriophyllo-Nupharetum lutei. Por otra parte, en el centro Peninsular diferentes autores (Arnaiz y Molina, 1986; Loidi Arregui et al., 1997) han reconocido comunidades de Polygonum amphibium como tales. El grupo aquí estudiado posee diferencias florísticas y 

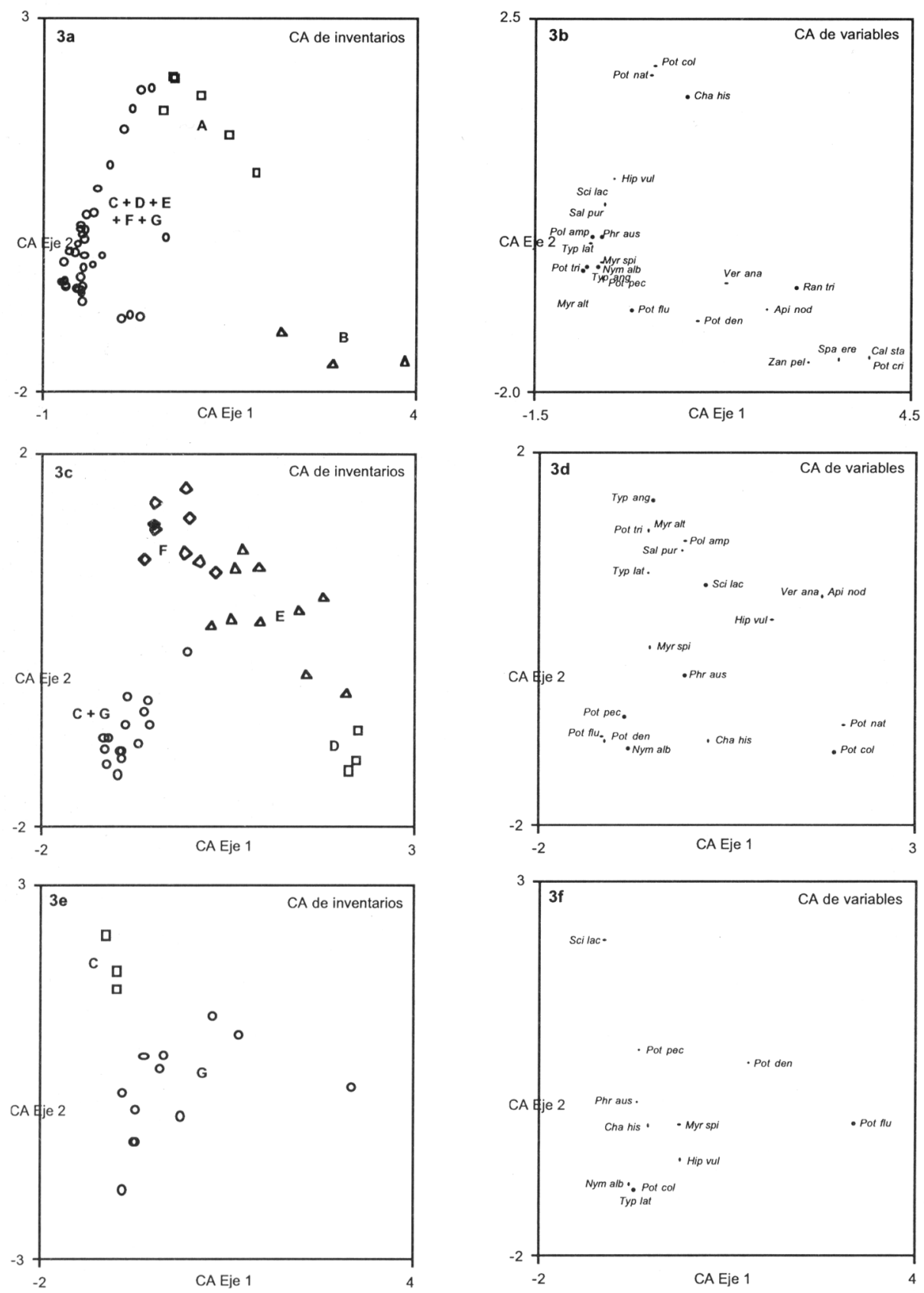

Figura 3. Ordenación mediante análisis de correspondencias de: todos los inventarios (3a, 3b); los grupos C, D, E, F y G (3c, 3d); los grupos C y G (3e, 3f). Grupos: A, Comunidad de Chara hispida; B, Groenlandio densae-Zannichellietum peltatae; C, Potamo pectinati-Myriophylletum spicati; D, Potametum colorati; E, Hippuridetum vulgaris; F, Comunidad de Potamogeton amphibium; G, Myriophyllo-Nupharetum lutei. $C A$ ordinations of the all relevés $(3 a, 3 b) ; C A$ ordinations of the groups $C, D, E, F$ and $G(3 c, 3 d) ; C A$ ordinations of the groups $C$ and $G(3 e, 3 f)$. 
ecológicas suficientes para justificar su independencia del grupo siguiente.

El grupo G (tab. 2, inv. 39-50) reúne todos aquellos inventarios dominados o codominados por Myriophyllum spicatum con un estrato natante de ninfeidos (Nymphaea alba) o sin él. El subgrupo carente de Nymphaea alba (inv. 44-50) es florísticamente homogéneo pero ecologicamente heterogéneo ya que reúne inventarios tanto de ambientes lénticos (inv. 44-47) como lóticos (48-50). Sintaxonómicamente, este grupo se ha incluido en la asociación Myriophyllo-Nupharetum lutei.

\section{Ordenación}

En las figuras 3 a y 3 b se observa que el grupo B se separa hacia el extremo inferior derecho del diagrama debido a la presencia de Ranunculus trichophyllus, Groenlandia densa, Zannichellia peltata, Potamogeton crispus, Callitriche stagnalis, Sparganium erectum y Apium nodiflorum, que indican, en general, aguas someras y eutrofizadas. El grupo A se separa hacia la zona central-izquierda del eje 1 y superior del eje 2 en base a tres plantas: Chara hispida, Potamogeton coloratus y Potamogeton natans, de aguas profundas y oligótrofas. En el extremo izquierdo del diagrama se reúnen los grupos C, D, E, F y G. Las figuras $3 \mathrm{c}$ y $3 \mathrm{~d}$ muestran un análisis de correspondencias de la matriz obtenida con estos cinco grupos. En ellas, el grupo D se separa hacia el extremo inferior derecho del diagrama, debido a la presencia de Potamogeton coloratus y Potamogeton natans. El grupo E se separa hacia el centro-derecha del eje 1 y la zona centro-superior del eje 2 en base a la presencia de Hippuris vulgaris, de aguas básicas más eútrofas. El grupo $\mathrm{F}$ se separa hacia el centro-izquierda del eje 1 y la zona superior del eje 2 debido a plantes tales como Myriophyllum alterniflorum y Potamogeton trichoides, de aguas menos básicas. En el cuadrante inferior izquierdo quedan entremezclados los grupos C y G. Las figuras 3e y 3 f muestran un análisis de correspondencias de la matriz obtenida con estos dos grupos. En ellas, el grupo $\mathrm{G}$ se separa en base a la presencia de plantas como Myriophyllum spicatum, Nymphaea alba, de aguas quietas y profundas. El grupo C, a pesar de compartir Potamogeton pectinatus con el grupo $\mathrm{G}$, se separa hacia el cuadrante superior izquierdo del diagrama, debido a la ausencia del resto de los táxones presentes en este último grupo como resultado de las condiciones reófilas que caracterizan el hábitat de este grupo.

\section{Esquema sintaxonómico}

1. CHARETEA FRAGILIS Fukarek ex Krausch 1964

+ Charetalia hispidae Sauer ex Krausch 1964

* Charion canescentis Krausch 1964

1. Comunidad de Chara hispida

2. POTAMETEA Tüxen \& Preising 1942

+ Potametalia W. Koch 1926

* Potamion W. Koch 1926

2. Groenlandio densae-

Zannichellietum peltatae Velayos, Carrasco y Cirujano 1989

3. Potamo pectinati-Myriophylletum spicati Rivas Goday 1964 corr. Conesa 1990

4. Potametum colorati Allorge 1922

5. Hippuridetum vulgaris Passarge 1955

6. Comunidad de Potamogeton amphibium

* Nymphaeion albae Oberdorfer 1957

7. Myriophyllo-Nupharetum lutei W. Koch 1926 


\section{BIBLIOGRAFÍA}

ALONSO, M. - 1987-Clasificación de los complejos palustres españoles. Seminario sobre Bases Científicas para la protección de los humedales en España. Publ. Real Acad. Ci. Exact., 65-78.

ARNAIZ, C. y J.A. MOLINA -1986- Vegetación acuática y helofítica de la cuenca alta del río Guadarrama (Madrid, España). Lazaroa 8: 221-240.

ASENSI, A. y J.M. NIETO -1981- Vegetación acuática, halófila y halonitrófila de la provincia de Málaga. Trab. Monogr. Dep. Bot. Málaga 2: 105-122.

BIURRUN GALARRAGA, I. -1995- Flora y vegetación acuática, higrófila y halófila de las cuencas de los ríos Arga y Bidasoa en Navarra. Mem. Doctoral, Fac. Ciencias Biológicas, Univ. Pais Vasco.

BRAUN-BLANQUET, J. -1979- Fitosociología. Bases para el estudio de las comunidades vegetales. Ed. H. Blume, Madrid, 820 pp.

BUENO SÁNCHEZ, A. -1997- Flora y vegetación de los estuarios asturianos. Ed. Servicio Central de Publicaciones del Principado de Asturias, Cuadernos de Medio Ambiente, Naturaleza 3, Oviedo, $352 \mathrm{pp}$.

CARSTENSEN, U. - 1955- Laichkrautgesellschaften an Kleingewässern Schleswig-Holsteins. Schriften Naturwiss. Vereins SchleswigHolstein 27(2): 144-170.

CIRUJANO, S. - 1980- Las lagunas manchegas y su vegetación I. Anales Jard. Bot. Madrid 37 (1): 155-192.

COSTA, M., H. BOIRA, J.B. PERIS y G. STÜBING -1986- La vegetación acuática y palustre valenciana. Ecol. Medit. 12(1-2): 83-100.

FONT QUER, P. -1982-Diccionario de Botánica. Ed. Labor, S.A., Barcelona, 1244 pp.

HARTOG, C. DEN \& S. SEGAL -1964- A new classification of the water plant communities. Acta Bot. Neerl. 13: 367-393.

LOIDI ARREGUI, J., I. BIURRUN GALARRAGA, y M. HERRERA GALLASTEGUI -1997- La vegetación del centro-septentrional de España. Itinera Geobot. 9: 161-618.

MERIAUX, J.L. - 1978-Etude Analytique et Comparative de la Végétation Aquatique d'Etangs et Marais du Nord de la France (Vallée de la Sensée et Bassin Houillier du Nord-Pas-de-Calais). Documents Phytosociol. N.S. 3: 1-244.
MOLINA, J.A. -1996- Sobre la vegetación de los humedales de la Península Ibérica (1. PhragmitiMagnocaricetea). Lazaroa 16: 27-88.

MUELLER-DOMBOIS, D. \& H. ELLENBERG 1974- Aims and methods of vegetation ecology. J. Wiley and Sons, New York.

PEINADO LORCA, M. y J.M. MARTÍNEZ PARRAS -1987- Castilla-La Mancha. In Peinado Lorca, M. y Rivas-Martínez, S. (Eds.) $L a$ Vegetación de España. Universidad de Alcalá de Henares, 544 pp.

PENAS MERINO, A. y T. DÍAZ GONZÁLEZ 1985- Hippuris vulgaris L. en la Península Ibérica. Anales Jard. Bot. Madrid 41(2): 456457.

PÉREZ RAYA, F. y J.M. LÓPEZ NIETO -1991Vegetación acuática y helofítica de la Depresión del Padul (Granada). Acta Bot. Malacitana 16(2): 373-389.

PHILIPPI, G. -1974- Phragmitetea Tx. et Prsg. 42. In Oberdorfer, E. -1977- Süddeutsche Pflanzengesellschaften, 1. Gustav Fischer Verlag Jena, $311 \mathrm{pp}$.

PODANI, J. -1989- New combinatorial clustering methods. Vegetatio, 81: 61-77.

PODANI, J. -1993-sYN-TAX-pc. Computer Programs for Multivariate Data Analysis in Ecology and Systematics. Version 5.0. Scientia Publishing, Budapest.

POTT, R. -1992- Die Pflanzengesellschaften Deutschlands. Verlag Eugen Ulmer Stuttgart, $427 \mathrm{pp}$.

RIVAS GODAY, S. -1964-Vegetación y flórula de la cuenca extremeña del Guadiana. Publ. Excma. Diputac. Badajoz, Madrid, 799 pp.

RIVAS-MARTÍNEZ, S. -1987-Mapa y Memoria de las series de vegetación de España 1: 400.000. ICONA, Madrid, $268 \mathrm{pp}$.

RIVAS-MARTÍNEZ, S., M. COSTA, S. CASTROVIEJO y E. VALDÉS -1980Vegetación de Doñana (Huelva, España). Lazaroa 2: 3-189.

RIVAS-MARTÍNEZ, S., P. CANTÓ, F. FERNÁNDEZ-GONZÁLEZ, C. NAVARRO, J.M. PIZARRO y D. SÁNCHEZ-MATA -1990Biogeografía de la Península Ibérica, Islas Baleares y Canarias. Folia Bot. Matritensis 8: 1-6.

RODRIGUEZ OUBIÑA，J. -1986- Estudio fitosociológico de las Brañas de la provincia de 
A Coruña. Mem. Doctoral Universidad de Santiago.

SNEATH, P. \& R. SOKAL -1973- Numerical Taxonomy. Freeman, San Francisco, CA.

TER BRAAK, C.J.F. -1985- Canoco-a fortran program for community ordination by [partial] [detrended] [canonical] correspondence analysis, principal components analysis and redundance analysis. Version 2.1 ITI-TNO, Wageningen, The Netherlands.

TER BRAAK, C.J.F. -1990- Update notes: CANOCO version 3.10 Agricultural Mathematics Group, Wageningen, The Netherlands.

TUTIN, T.G. et al. (Eds.) -1964/1980- Flora Europaea 1-5. Cambridge University Press, Cambridge.

VAN DER MAAREL, E. -1979- Transformation of cover-abundance values in phytosociology and its effects on community similarity. Vegetatio 39: 97-114.

VELAYOS, M., S. CIRUJANO y A. MARQUINA 1984- Aspectos de la vegetación acuática de la provincia de Guadalajara. Anales Jard. Bot. Madrid 41(1): 175-184.
VELAYOS, M., M.A. CARRASCO y S. CIRUJANO -1989- Las lagunas del Campo de Calatrava (Ciudad Real). Bot. Complutensis 14: 9-50.

WESTHOFF, V. \& E. VAN DER MAAREL -1978The Braun-Blanquet approach. In: Whittaker, R.H. (Ed.) Classification of plant communities, 287-399. Junk, Den Haag.

Aceptado para su publicación en Agosto de 1998

Dirección de los autores. J. A. MOLINA: Departamento de Biología Vegetal II, Facultad de Farmacia, Universidad Complutense de Madrid, E28040 Madrid (España). S. SARDINERO: Instituto Nacional de Investigaciones Agrarias, Centro de Investigaciones Forestales, ctra. N-VI km 7, E28040 Madrid (España). 